\title{
Qualitative Modeling and Simulation of Bacterial Regulatory Networks
}

\author{
Hidde de Jong \\ INRIA Grenoble-Rhône-Alpes \\ 655 Avenue de l'Europe, Montbonnot, 38334 Saint-Ismier Cedex, France \\ Hidde.de-Jong@inria.fr
}

The adaptation of microorganisms to their environment is controlled at the molecular level by large and complex networks of biochemical reactions involving genes, RNAs, proteins, metabolites, and small signalling molecules. In theory, it is possible to write down mathematical models of these networks, and study these by means of classical analysis and simulation tools. In practice, this is not easy to achieve though, as quantitative data on kinetic parameters are usually absent for most systems of biological interest. Moreover, the models consist of a large number of variables, are strongly nonlinear and include different time-scales, which make them difficult to handle both mathematically and computationally.

We have developed methods for the reduction and approximation of kinetic models of bacterial regulatory networks to simplified, so-called piecewise-linear differential equation models. The qualitative dynamics of the piecewise-linear models can be studied using discrete abstractions from hybrid systems theory. This enables the application of model-checking tools to the formal verification of dynamic properties of the regulatory networks. The above approach has been implemented in the publicly-available computer tool Genetic Network Analyzer (GNA) and has been used to analyze a variety of bacterial regulatory networks.

I will illustrate the application of GNA by means of the network of global transcription regulators controlling the adaptation of the bacterium Escherichia coli to environmental stress conditions. Even though E. coli is one of the best studied model organisms, it is currently little understood how a stress signal is sensed and propagated through the network of global regulators, and leads the cell to respond in an adequate way. Qualitative modeling and simulation of the network of global regulators has allowed us to identify essential features of the transition between exponential and stationary phase of the bacteria and to make new predictions on the dynamic behavior following a carbon upshift. 